\title{
Integrable solutions of a functional-integral equation
}

\author{
JOZEF BANAS and ZYGMUNT KNAP
}

\begin{abstract}
This paper contains a theorem on the existence of monotonic and integrable solutions of a functional-integral equation. The proof of that theorem is based on the technique associated with the notion of a measure of weak noncompactness.
\end{abstract}

\section{INTRODUCTION}

Integral equations of various types play very significant role in many branches of linear and nonlinear functional analysis and their applications. For instance, the most frequently investigated integral equations are the Fredholm linear equation or its nonlinear counterparts, the Hammerstein and Urysohn integral equations (cf. $[1,7,12,21,22]$ ). Apart from the above mentioned there are also considered other types of integral equations, mostly in connection with functional equations. Functional-integral equations of such a type have been studied in several papers and monographs $[3,9,12,14$, $15,21]$ and create also the main subject of the present paper.

Roughly speaking, our considerations were initiated by the paper [3], where the functional-integral equations of the convolution type were investigated. Equations of this type and the equation(1) considered in the sequel, appear often in many applications. For example, they can be applied in solving many problems in physics, engineering and economics (cf. [9,21] and references given there). Also a lot of problems considered in the theory of partial differential equations lead us to functional-integral equations of the type (1) $[14,17,18]$.

It is worthwhile to mention that the results in the paper [3] have been obtained with help of the complicated theory of monotone and accretive operators while we use the fixed point theorem due to Emmanuele. This

1980 Mathematics Subject Classification (1985 revision); 47 H 15.

Editorial de la Universidad Complutense. Madrid, 1989. 
theorem allows us to prove more general existence result in rather simple way.

\section{NOTATION, DEFINITIONS AND AUXILIARY FACTS}

This section is devoted to recall some auxiliary results which will be needed further on.

Let $L^{1}(a, b)$ denote the space of Lebesgue integrable functions on the interval $[a, b]$, with the standard norm

$$
\|x\|=\int_{a}^{b}|x(t)| d t .
$$

For simplicity, we shall consider the space $L^{1}=L^{1}(0,1)$.

Assume that $f(t, x)=f:[0,1] \times R \rightarrow R$ satisfies Carathéodory conditions, i.e. it is measurable in $t$ for any $x$ and continuous in $x$ for almost all $t$. Then to every function $x(t)$ being measurable on the interval $[0,1]$ we may assign the function $(F x)(t)=f(t, x(t)), t \in[0,1]$. The operator $F$ defined in such a way is called the superposition operator. Notice that this operator is one of the simplest and most important operator investigated in nonlinear functional analysis (cfr. $[1,12,21,22]$ ).

We have the following theorem due to Krasnosel'skii [1].

Theorem 1. The superposition operator $F$ maps continuously the space $L^{\prime}$ into itself if and only if $|f(t, x)| \leq a(t)+b|x|$ for all $t \in[0, I]$ and $x \in R$, where $a(t)$ is a function from $L^{1}$ and $b$ is a nonnegative constant.

Now, let $E$ denote an arbitrary Banach space and $X$ be a nonempty and bounded subset of $E$. Moreover, denote by $K_{r}$ the closed ball in $E$ centered at $\theta$ and with radius $r$.

Let us recall the notion of the measure of weak noncompactness defined by De Blasi [6] in the following way

$$
\beta(X)=\inf \left[r>0: \text { there exists a weakly compact subset } \begin{array}{r}
Y \text { of } E \text { such that } \\
X \subset Y+K_{r}
\end{array}\right] .
$$

The function $\beta(X)$ possesses several useful properties which may be found in [6] (cf. also [5], where an axiomatic approach to the notion of a measure of weak noncompactness is presented). 
The convenient and handy formula for the function $\beta(X)$ in the space $L^{1}$ was given recently by Appell and De Pascale [2] (cf, also [5]):

$$
\beta(X)=\lim _{\epsilon \rightarrow 0}\left\{\sup _{x \in X}\left\{\sup \left[\int|x(t)| d t: D \subset[0,1], \text { meas } D \leq \epsilon\right]\right\}\right\},
$$

where the symbol meas $D$ stands for Lebesgue measure of a set $D$.

The applicability of the measure of weak noncompactness $\beta$ was pointed out by Emmanuele [8] who proved the following fixed point theorem.

Theorem 2. Let $Q$ be a nonempty, closed, convex and bounded subset of $E$ and let $T: Q \rightarrow Q$ be a weakly continuous operator (i.e. mapping weakly convergent sequences into itself) having the property that there is a constant $k \in[0,1)$ such that

$$
\beta(T X) \leq k \beta(X)
$$

for any nonempty subset $X$ of $Q$. Then $T$ has at least one fixed point in the set $Q$.

It is worthwhile to mention that weak continuity of an operator is rather difficult to check in a concrete situation $[7,16]$. For subsequent purposes we describe now the example of a set in the space $L^{1}$ which allows us to overcome the above mentioned difficulty.

For $r>0$ denote by $Q_{r}$ the subset of the ball $K_{r}$ in the space $L^{1}$ consisting of all functions which are a.e. nondecreasing or nonincreasing on the interval $[0,1]$. Obviously the set $Q_{r}$ is nonempty, bounded, convex and closed in $L^{1}$. In addition, we have the following.

Theorem 3. Any continuous operator $S: Q_{r} \rightarrow L^{1}$ is weakly continuous.

For the proof we refer to [4].

\section{MAIN RESULT}

We shall consider the following functional-integral equation

$$
x(t)=g(t)+f\left(t, \int_{0}^{1} k(t, s) x(\varphi(s)) d s\right), t \in[0,1]
$$


For convenience the operator

$$
(H x)(t)=f\left(t, \int_{0}^{1} k(t, s) x(s) d s\right)
$$

will be written as the product $H=F K$ of the linear integral operator

$$
(K x)(t)=\int_{0}^{1} k(t, s) x(s) d s
$$

and the superposition operator

$$
(F x)(t)=f(t, x(t)) .
$$

Thus the equation (1) has the form

$$
x=A x=g+H x(\varphi)=g+F K x(\varphi) .
$$

Now we formulate the assumptions under which the equation (1) will be investigated. Namely, we assume the following:

(i) $g \in L^{1}$ and is a.e. nonincreasing and positive on the interval $[0,1]$,

(ii) $f:[0,1] \times R \rightarrow R_{+}=[0,+\infty)$ satisfies Carathéodory conditions and there exist a function $a \in L^{1}$ and a constant $b \geq 0$ such that

$$
f(t, x) \leq a(t)+b|x|
$$

for all $t \in[0,1]$ and $x \in R$. Moreover, $f(t, x)$ is assumed to be non-increasing on the set $[0,1] \times R$ with respect to $t$ and nondecreasing with respect to $x$,

(iii) $k:[0,1] \times[0,1] \rightarrow R_{+}$is measurable with respect to both variables and such that the integral operator $K$ (defined above) maps $L^{\prime}$ into itself.

Let us recollect that the assumption (iii) implies [12] that the operator $K$ maps continuously the space $L^{1}$ into $L^{1}$. From now on by $\|K\|$ we shall denote the norm of the operator $K$.

Apart from this we assume.

(iv) for every $p \in[0,1]$ and for all $t_{1}, t_{2} \in[0,1]$ the following condition holds true

$$
t_{1}<t_{2} \Rightarrow \int_{0}^{p} k\left(t_{1}, s\right) d s \geq \int_{0}^{p} k\left(t_{2}, s\right) d s,
$$


(v) $\varphi:[0,1] \rightarrow[0,1]$ is increasing, absolutely continuous and there exists a constant $B>0$ such that $\varphi^{\prime}(t) \geq B$ for almost all $t \in[0,1]$,

(vi) $b\|K\| / B<1$.

Then we can prove the following theorem.

Theorem 4. Under the above assumptions the equation (1) has at least one solution $x \in L^{1}$ which is a.e. nonincreasing on the interval $[0,1]$.

Proof. Take an arbitrary $x \in L^{\prime}$. In virtue of the assumptions (i), (ii), (iii) and $(v)$ it is easily seen that $A x \in L^{1}$, where $\mathrm{A}$ is the operator defined by (2). Moreover, we get

$$
\begin{gathered}
\|A x\| \leq\|g\|+\|F K x(\varphi)\| \leq\|g\|+\int_{0}^{1}\left[a(s)+b \mid \int_{0}^{1} k(s, \tau) x(\varphi(\tau)) d \tau \|\right] d s \leq \\
\leq\|g\|+\|a\|+b\|K\|\|x(\varphi)\| \leq\|g\|+\|a\|+(b\|K\| / B) \int_{0}^{1}|x(\varphi(s))| \varphi^{\prime}(s) d s \leq \\
\leq\|g\|+\|a\|+(b\|K\| / B)\|x\| .
\end{gathered}
$$

The above estimate yields that the operator A maps the ball $K_{r}$ into itself, where $r=(\|g\|+\|a\|) /\left(1-b\|K\| B^{-1}\right)$.

Now let $Q_{r}$ denote the subset of $K_{r}$ consisting of all functions being positive and a.e. nonincreasing on $[0,1]$. Obviously $Q_{r}$ is nonempty, bounded, closed and convex (compare Section 2). Let us take an arbitrary function $x \in Q_{r}$. Then $x(\varphi)$ is a.e. nonincreasing and positive on the interval $[0,1]$. Its image $K x(\varphi)$ is also of the same kind. This assertion can be derived from the result due to Krzyż [13] if we take into account the assumption (iv) and the part of assumption (iii) saying that $k(t, s) \geq 0$ for $(t, s) \in[0,1]^{2}$. Further, in view of the assumptions (ii) and (v) we deduce that $A x$ is a.e. nonincreasing and positive on the interval $[0,1]$. Thus, keeping in mind that A: $K_{r} \rightarrow K_{r}$ we conclude that A is a selfmapping of the set $Q_{r}$.

Now, let us observe that the assumptions (iii) and (ii) imply that $\mathrm{A}$ is continuous on the set $Q_{r}$ (compare Theorem I). In the light of Theorem 3 this allows us to infer that A is weakly continuous on the set $Q_{r}$.

In what follows take a nonempty set $X \subset Q$, and fix $\epsilon>0$. Further, let $D \subset[0,1]$ be such that meas $D \leq \epsilon$ : Then, for an arbitrary $x \in X$ in view of our assumptions we obtain

$$
\begin{aligned}
\int_{b}|(A x)(t)| d t & \leq \int_{D}|g(t)| d t+\int_{D} a(t) d t+b \int_{b}\left|\int_{0}^{1} k(t, s) x(\varphi(s)) d s\right| d t= \\
& =\|g\|_{L^{\prime}(D)}+\|a\|_{L^{\prime}(D)}+b\|K x(\varphi)\|_{L^{\prime}(D)} .
\end{aligned}
$$


Next, calling on the fact that the operator $K$ maps the space $L^{\prime}(D)$ into itself and is continuous, we get

$$
\int_{f}|(A x)(t)| d t \leq\|g\|_{L^{\prime}(D)}+\|a\|_{L^{\prime}(D)}+b\|K\|_{D}\|x(\varphi)\|_{L^{\prime}(D)},
$$

where the symbol $\|K\|_{D}$ stands for the norm of the operator $K \cdot L^{\prime}(D) \rightarrow L^{1}(D)$.

Consequently, we have

$$
\int_{D}|(A x)(t)| d t \leq\|g\|_{L^{\prime}(D)}+\|a\|_{L^{\prime}(D)}+\left(b\|K\|_{D} / B\right) \int_{b}|x(\varphi(t))| \varphi^{\prime}(t) d t .
$$

Now, applying the theorem on integration by substitution for Lebesgue integrals we can rewritten the last estimate in the following way

$$
\int_{D}|(A x)(t)| d t \leq\|g\|_{L^{\prime}(D)}+\|a\|_{L^{\prime}(D)}+\left(b\|K\|_{D} / B\right) \int_{\varphi(D)}|x(t)| d t .
$$

Further, taking into account the obvious equality

$$
\lim _{\epsilon \rightarrow 0}\left\{\sup \left[\int g(t) d t+\int_{D} a(t) d t: D \subset[0,1] \text {, meas } D \leq \epsilon\right]\right\}=0
$$

and keeping in mind that the function $\varphi$ is supposed to be absolutely continuous we obtain

$$
\beta(A X) \leq(b\|K\| / B) \beta(X) .
$$

This inequality together with earlier indicated properties of the operator A

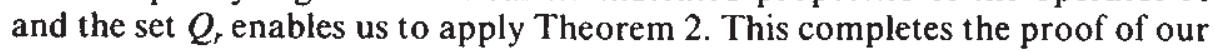
theorem.

\section{FINAL REMARKS AND COMMENTS}

In this section we give a few comments concerning the assumptions of Theorem 4.

At first let us notice that it is very difficult to find necessary and sufficient conditions for the function $k(l, s)$ guaranteeing that the linear operator $K$ generated by this function maps $L^{1}$ into $L^{1}$. In some special cases this problem has been solved by Tomaselli [20]; see also [19] and [10] for further extensions of the result due to Tomaselli. Some other special cases are also discussed in [21]. 
It is worthwhile to mention the following sufficient condition guaranteeing that $K: L^{1} \rightarrow L^{1}$ and is continuous [7]:

The function $k:[0,1] \times[0,1] \rightarrow R$ is measurable and there exists a positive constant $M$ such that

$$
\begin{aligned}
& \text { ess sup } \int_{0}^{1}|k(t, s)| d s \leq M, \\
& t \in[0,1]{ }_{0} \\
& \text { ess sup } \int_{s \in[0,1]}^{1}|k(t, s)| d t \leq M,
\end{aligned}
$$

The above also imply that the norm of the operator $K$ is majorized by $M$, i.e. $\|K\| \leq M$.

Let us notice that the above condition seems to be not very convenient in practise because it is rather difficult to verify if it holds in a concrete situation.

Secondly, let us observe that the assumption on the positiveness of the functions involved (i.e. $g, f$ and $k$ ) may be omitted if we assume the following condition to be satisfied

$$
\text { (vii) } \quad \int_{0}^{1} k(t, s) d s=\text { const, for each } t \in[0,1] \text {. }
$$

Indeed, the above assumption together with assumption (iv) imply that the integral operator $K$ transforms monotonic functions into monotonic ones [13]. Thus in the proof of Theorem 4 we may consider instead of $Q_{r}$ the set of all functions $x \in K_{r}$ being a.e. nonincreasing on the interval [0,1] (but not necessarily positive). Obviously the reminder of the proof will be quite analogous as previously.

On the other hand the condition (vii) seems to be too restrective.

Finally let us mention that up to now existence theorems for the equations like to (1) have been proved under more restrective assumptions than those made here. For example, it was assumed that $F$ is the so-called "improving" operator (cf. [12, 21, 22]). An assumption of such a kind has also been made in the paper [3].

\section{References}

[1] J. APPELL, On the solvability of nonlinear noncompact problems in function spaces with applications to integral and differential equations, Boll. Un. Mat. Ital. (6), 1-B (1982), I161-1177. 
[2] J. APPELL and E. DE PASCALE, Su alcuni parametri connesi con la misura di non compattezza di Hausdorff in spazi di functioni misurabili, Boll. Un. Mat. Ital. (6), 3-B (1984), 497-515.

[3] S. N. ASKHABOV and K. S. MUKHTAROV, On a class of nonlinear integral equations of convolution type, Differentsial'nye Uravneniya 23 (1987), 512-514 (in Russian).

[4] J. BANAS, Integrable solutions of Hammerstein and Urysohn integral equations, J. Austral. Math. Soc. 45 (1988). (to appear).

[5] J. BANAS and J. RIVERO, On measures of weak noncompactness, Ann. Mat. Pura Appl. 151 (1988), 213-224.

[6] F. S. DE BLASI, On a property of the unit sphere in a Banach space, Bull. Math. Soc. Sci. Math. R. S. Roumanie 21 (1977), 259-262.

[7] N. Dunford and J. SChwarTZ, Linear Operators I, Int. Publ., Leyden 1963.

[8] G. EMMANUELE, Measure of weak noncompactness and fixed point theorems, Bull. Math. Soc. Sci. Math. R. S. Roumanie 25 (1981), 253-258.

[9] J. K. HALE, Theory of functional differential equations, Springer 1977.

[10] R. K. JUBERG, Measure of noncompactness and interpolation of compactness for a class of integral transformations, Duke Math. J. 41 (1974), 511-525.

[11] M.A. KRASNOSEL'SKII, On the continuity of the operator $F u(x)=f(x, u(x))$, Dokl. Akad. Nauk SSSR 77 (1951), 185-188 (in Russian).

[12] M. A. Krasnosel'skit, P. P. Zabrejko, J. I. Pustyl'ilk and P. J. SoboLEVSK11, Integral operators in spaces of summable functions, Nauka, Moscow 1966 [English translation: Noordhoff, Leyden 1976].

[13] J. KRZYZ, on monotonity-preserving transformations, Ann. Univ. Marie CurieSkTodowska, 6, Sect. A (1952), 91-111.

[14] M. KWAPISZ and J. TURO, On the existence and uniqueness of solutions of Darboux problem for partial differential-integral equations, Colloq. Math. 29(1974), 295-318.

[15] M. KWAPISZ and J. TURo, Some integral-functional equations, Funkcial. Ekvac. 18 (1975), 107-162.

[16] V. LAKSHMIKANTHAM and S. LEELA, Nonlinear differential equations in abstract spaces, Pergamon Press, New York, 1981.

[17] K. NowICKA, On the existence of solutions for some integral-functional equation, Comment. Math. 23 (1983), 279-293.

[18] A. PElCZAR, Some functional differential equations, Dissert. Math. 100 (1973), 3-110.

[19] C. A. STUART, The measure of noncompactness of some linear integral operators, Proc. Roy. Soc. Edinburgh 71 (1973), 167-179.

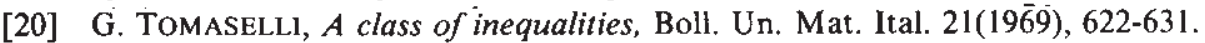

[21] P. P. Zabrejko, A. I. Koshel.ev, M. A. Krasnosel.Skil, S. G. Mikhlin, L. S. RAKovSHCHIK and V. J. STETSENKo, Integral equations, Nauka, Moscow 1968 [English translation: Noordhoff, Leyden 1975].

[22] P. P. ZABREJKo and PUSTYL'NIK, On the continuity and complete continuity of nonlinear integral operators in $L^{p}$ spaces, Uspehi Mat. Nauk 19 (1964), 204-205 (in Russian).

Departament of Mathematics

Technical University of Rzeszow

35-959 Rzeszów, W. Pola 2

Poland

Recibido: 29 de abril de 1988 